\title{
CONCIENCIA PRE-REFLEXIVA Y LA TESIS DE LA IDENTIDAD EXPERIENCIA/EXPERIMENTADOR
}

\author{
PRE-REFLECTIVE CONSCIOUSNESS AND THE EXPERIENCE/EXPERIENCER \\ IDENTITY THESIS
}

\author{
Esteban Ortiz Medina* \\ Universidad de Buenos Aires - IIF-SADAF-CONICET, \\ Buenos Aires - Argentina. \\ Recibido mayo de 2019/Received May, 2019 \\ Aceptado agosto de 2019/Accepted August, 2019
}

\begin{abstract}
RESUMEN
Una de las críticas que se les realizan a las teorías no-egológicas de la conciencia es que negarían al sujeto de experiencia al nivel de la conciencia fenoménica. Pero claramente lo anterior no puede ser. La conciencia fenoménica siempre es para un sujeto de experiencia. Justamente de eso se trata ésta. Por ello la idea de este trabajo es que se puede estipular que la conciencia posee una estructura no-egológica sin que ello implique negar la existencia del sujeto de experiencia. No hay que confundir al Ego con el sujeto de experiencia. Es por esto, es que se hará revisión de la tesis de la identidad experiencia/experimentador. Según la cual, y desde elementos auto-representacionales, la experiencia se toma a sí misma como sujeto de experiencia. Así, la experiencia representándose a sí misma sería su propio sujeto de experiencia. La experiencia representándose a sí misma sería idéntica a su propio sujeto de experiencia.

Palabras Clave: Teorías no-egológicas de la conciencia, Conciencia pre-reflexiva, Auto-representación, Tesis de la identidad experiencia/experimentador.
\end{abstract}

\begin{abstract}
One of the critiques to the non-egological theories of consciousness is that they would deny the subject of experience at the level of phenomenal consciousness. But clearly this cannot be. Phenomenal consciousness is always for a subject of experience. That's exactly what this one's about. Therefore, the idea of this work is that it is possible to stipulate that consciousness has a non-egological structure without denying the existence of the subject of experience. We must not confuse the Ego with the subject of experience. This is why we will revise the experience/experiencer identity thesis. According to which, and from self-representational elements, experience takes itself as the subject of experience. Thus, experience representing itself would be its own subject of experience. The experience representing itself would be identical to its own subject of experience.

Key Words: Non-egological theories of consciousness, Pre-reflexive consciousness, Self-representation, Experience/ Experiencer Identity Thesis.
\end{abstract}

* Autor correspondiente / Corresponding author: edeoeme@ gmail.com 


\section{PRELIMINARES}

Dentro de los problemas concernientes a la conciencia fenoménica, existe una discusión sobre la estructura de la conciencia ${ }^{1}$. De si ésta posee o no una estructura egológica. Este problema surge porque la conciencia fenoménica posee dos componentes. El carácter cualitativo, el "algo que es cómo es", y el carácter subjetivo, el "ser para un sujeto (o para mí)". El carácter cualitativo es el factor que explica la variedad de los estados mentales conscientes, explicaría la diferencia entre la experiencia consciente de mirar una raíz de castaño y la experiencia consciente de mirar un partido de fútbol. Las experiencias conscientes poseen diferencias cualitativas porque representan distintas sumas de propiedades cualitativas del ambiente en un tiempo dado. El carácter subjetivo es el factor común a todo estado mental consciente, que sería un cierto matiz subjetivo. El carácter cualitativo de un estado consciente define qué tipo de estado consciente es (sus características); en cambio el carácter subjetivo de un estado consciente es precisamente lo que lo hace ser un estado consciente como tal. Esto quiere decir que hay algo que es cómo es ser para un sujeto encontrarse en un determinado estado mental. Todo estado mental consciente tendría un cierto carácter subjetivo. Todo estado mental consciente ocurriría en alguien pero también, y esto es lo importante, ocurriría para alguien, es decir, alguien tiene que estar consciente del estado mental como suyo. Lo cual da pie para sostener que la conciencia posee una estructura egológica o, mejor dicho, pareciese que existe una conexión entre el carácter subjetivo y una estructura egológica de la conciencia. Es decir, el Ego (o Yo) sería el sujeto de experiencia.

Por estructura egológica de la conciencia habrá que entender, mínimamente, que hay un Ego/Yo presente en la conciencia, de tal manera que una persona es consciente de que ella misma se encuentra en ese estado. Así, la conciencia fenoménica tendría la siguiente forma: "YO me encuentro en un estado mental M ahora". En rigor, una teoría egológica de la conciencia, por un lado, postularía la existencia de una entidad (en tanto sujeto de experiencia) numéricamente distinta de los mentales fenoménicamente conscientes que sea capaz de instanciarlos y así dar cuenta del contenido de éstos. Y por el otro, aquella entidad, a su vez, es parte del contenido de los estados fenoménicamente conscientes ${ }^{2}$. Es decir, en una teoría egológica de la conciencia, el Ego/ Yo, a la vez, da cuenta y es parte del contenido de los estados fenoménicamente conscientes. Un ejemplo de estructura egológica de la conciencia se encuentra en la teoría de Gennaro $(2002)^{3}$.

Por estructura no-egológica se entenderá, mínimamente, lo siguiente, “(...) el Ego no está ni formalmente ni materialmente en la conciencia (...)." (Sartre, 1968, p. 11). Por lo tanto, su respuesta tiene que ser negativa a la pregunta: " ¿Existen el [Ego] como figurando en la (...) experiencia humana ordinaria?"' (Strawson, 1999, p. 118). Para estas teorías no se necesitaría de un Ego/Yo presente en la conciencia como contenido de la experiencia (salvo, quizá, en los estados mentales reflexivos), ni tampoco para dar cuenta del contenido de la experiencia. Es decir, para una teoría no-egológica de la conciencia no se necesita un Ego/Yo para dar cuenta de la funciones semánticas y meta-semánticas.

Las teorías no-egológicas de la conciencia, según Zahavi (2000, 2005, 2007, 2018), establecerían que es mejor referirse, a nivel de la conciencia fenoménica, como "hay conciencia de una silla" en vez de "Yo estoy viendo una silla". Esto traería como consecuencia que la conciencia sería anónima e impersonal. Lo cual significaría que habría experiencia pero no sujeto experimentador, es decir, las teorías no-egológicas de la conciencia negarían al sujeto de experiencia.

Claramente lo anterior no puede ser. Una experiencia consciente siempre es para un sujeto de experiencia. Justamente de eso se trata la conciencia fenoménica, hay algo que es cómo es para un sujeto de experiencia encontrarse en determinada experiencia consciente. Entonces Zahavi, ya que no se puede negar al sujeto de experiencia, concluiría que la conciencia tendría una estructura egológica.

$\mathrm{Si}$ bien creemos que es correcto que no se puede negar al sujeto de experiencia, también creemos que es incorrecto que, por no negar al sujeto de experiencia, se tenga que concluir que la conciencia tendría una estructura egológica. Una cosa no implica la otra. De eso se trata este trabajo. Perfectamente se puede estipular que la conciencia posee una estructura no-egológica sin que ello implique negar la existencia del sujeto de experiencia. No hay que confundir, como Zahavi lo hace, al Ego (o Yo) con el sujeto de experiencia. 
Para lograr mantener la existencia del sujeto de experiencia y una estructura no-egológica de la conciencia, es que se hará revisión de la tesis de la identidad experiencia/experimentador. La cual consiste en que la experiencia se toma a sí misma como sujeto de experiencia. Habría una identidad entre el sujeto de experiencia y su experiencia. Esto se lograría incluyendo elementos auto-representacionales en la conciencia. Con lo cual, la experiencia representándose a sí misma, refiriéndose a sí misma, sería su propio sujeto de experiencia. Con lo cual se presenta una tesis más económica. Puesto que, para dar cuenta del sujeto de experiencia, la tesis no-egológica de la conciencia no tiene la necesidad de postular otra entidad diferente del estado mental mismo.

\section{CONCIENCIA PRE-REFLEXIVA Y AUTO-REPRESENTACIÓN}

Para poder establecer lo anterior es necesario describir, primero, la conciencia pre-reflexiva ${ }^{4}$. La conciencia pre-reflexiva está compuesta por la conciencia posicional (o tética) y autoconciencia no-posicional (o no-tética) ${ }^{5}$. La conciencia es posicional en cuanto se trasciende para alcanzar un objeto, es decir, afirma o coloca la existencia del objeto intencional. Por conciencia o autoconciencia no-posicional se entiende la conciencia implícita (o periférica) de sí que acompaña a toda conciencia posicional ${ }^{6}$.

Pues bien, la conciencia pre-reflexiva no es más que el estado mental consciente compuesto por la autoconciencia no-posicional y la conciencia posicional. En efecto, dado que en primera instancia la conciencia se encuentra dirigida a otro objeto que no es ella, esto no basta para que sea completamente conciencia de tal objeto. Es necesario que a su vez sea autoconciencia no-posicional de sí misma. La autoconciencia no-posicional es constitutiva de la conciencia posicional de un objeto, tanto así que la conciencia posicional de un objeto no puede darse sin la autoconciencia no-posicional por la que un estado mental es consciente. Con lo cual, se puede llegar a la conclusión de que toda conciencia posicional de un objeto es a la vez conciencia no-posicional de sí misma ${ }^{7}$.

Es importante aclarar que si bien la conciencia pre-reflexiva está compuesta de la autoconciencia no-posicional y la conciencia posicional, eso no significa que sean dos estados mentales distintos, es decir, eso no significa que sean dos conciencias.
$\mathrm{Al}$ momento en que posicionalmente la conciencia pone el objeto se percata de sí misma no-posicionalmente en un único acto, que fenomenológicamente tiene una complejidad dual. Por lo tanto, no hay dos sino solo una conciencia. De ese modo, ahora se puede comprender la idea de que, por ejemplo, una conciencia de percibir se determina a la vez como conciencia de percibir y como percepción (Sartre, 1993).

La conciencia pre-reflexiva, entonces, es un solo estado mental consciente internamente diferenciado, el cual posee un elemento que está referido a sí mismo. Si la conciencia se entendiese como compuesta por dos estados mentales distintos, no se podría comprender la idea de que una conciencia de percibir se determina a la vez como conciencia de percibir y como percepción, como se dijo anteriormente. De modo que la conciencia de percibir sería algo que extrañamente se agregaría después, pues la percepción existiría "antes". Pero la conciencia de percibir es constitutiva de la percepción, como el modo mismo de su existencia, como la materia de que está hecho y no como una forma que se impondría con posterioridad a una materia (Sartre, 1993, p. 24).

En efecto, si la conciencia se entendiese como compuesta por dos estados mentales distintos, la conciencia no-posicional pasaría a ser una mera cualidad de la conciencia posicional. La conciencia posicional de algún estado mental tendría por añadidura, extrínsecamente, la cualidad de conciencia de sí. Por eso, en tanto que la conciencia pre-reflexiva es un solo estado internamente diferenciado que está referido a sí mismo, se puede caracterizar a esta conciencia como siendo un círculo no vicioso. Así su ser es indivisible e indisoluble ${ }^{8}$. Y si la conciencia de un estado mental no puede ser caracterizada como extrínseca entonces tiene que ser caracterizada como intrínseca (y auto-representacional).

Resulta importante aclarar la idea de caracterizar a la conciencia como intrínseca. En términos generales, la conciencia del estado mental es entendida como extrínseca cuando es enteramente distinta del estado mental, y la conciencia del estado mental es entendida como intrínseca cuando forma parte de un estado internamente diferenciado el cual se compone de la conciencia del estado mental y el estado mental. En otras palabras, la conciencia es intrínseca cuando ésta forma parte de un estado internamente diferenciado. 
Que la conciencia sea intrínseca toma fuerza de la siguiente intuición: hay una íntima relación que se establece entre la conciencia y aquello de lo que se es consciente. La idea de intimidad se entiende como que no hay una brecha entre cómo la experiencia consciente es y cómo la experiencia consciente parece que es. No es posible que, al tener una experiencia de una manzana roja, en donde el carácter cualitativo de esta experiencia es la rojez, el sujeto sea consciente de un carácter cualitativo verde. No es posible la brecha entre "las propiedades cualitativas que actualmente tiene la experiencia y las propiedades cualitativas de las que uno es consciente de tener" (Kriegel, 2009, p. 108).

La idea es que, al no haber la brecha comentada anteriormente, la conciencia y aquello de lo que uno es consciente no son totalmente distintos. Son parte del mismo estado mental internamente diferenciado. Entonces como consecuencia, de postular que la conciencia es intrínseca, se tiene la auto-representacionalidad de los estados mentales conscientes. Al aceptar que la conciencia es intrínseca a los estados mentales, “(...) la estructura de los estados conscientes incluye un elemento de auto-referencia" (Gennaro, 2012, p. 103). Los estados mentales conscientes tendrían "una especie de auto-referencialidad interna y un elemento relacional (...) dentro de la estructura de dichos estados" (Gennaro, 2012, p. 55). El elemento relacional es, más específicamente, "una relación interna intrínseca entre partes [de un estado complejo]" (Gennaro, 2012, p. 63). Dado que la conciencia que hace consciente al sujeto del estado mental es parte de este estado mental consciente, puede decirse que este estado se representa a sí mismo. Es decir, un estado mental consciente es un estado que posee la característica de que una parte intrínseca de sí mismo se encuentra dirigida hacia sí mismo. Hay, por tanto, una clara conexión entre intrínsecalidad y auto-representacionalidad ${ }^{9}$.

Con lo anterior es posible hacer la conexión con las teorías auto-representacionales de la conciencia (Kriegel, 2012a, 2012b, 2009). Las teorías auto-representacionales de la conciencia son un tipo de teoría intrínseca de la conciencia según la cual la conciencia de un estado mental es parte de ese estado mental. Además, al ser la conciencia de los estados mentales intrínseca, los estados mentales son representados por ellos mismos (es decir, se auto-representan), y al ser conscientes por lo tanto son representados por estados que son conscientes. Entones cuando se tiene una experiencia consciente del color rojo, se está consciente de la experiencia consciente, pero tal conciencia no es una conciencia extra que ocurre en adición a la experiencia. También es así porque la auto-representación de los estados mentales conscientes se da como conciencia periférica. Siguiendo con la experiencia del color rojo, se está focalmente consciente de la rojez, pero, a la vez, se está periféricamente consciente de la experiencia misma. Más específicamente, en este caso la conciencia periférica es conciencia periférica interna porque corresponde a la experiencia consciente misma, y la conciencia focal es conciencia focal externa porque corresponde al estado del mundo que representa la experiencia consciente ${ }^{10}$.

Siendo que la conciencia pre-reflexiva está compuesta de dos partes, es necesario aclarar qué tipo de relación mereológica es la que las partes pueden establecer entre sí. Es al tratar de responder esta cuestión que surge una distinción sobre el tipo de relación entre dichas partes: una mera suma de partes o un estado complejo de partes. Cuando se refiere a un estado complejo de partes, como es el caso de un estado mental consciente, estas partes están esencialmente interconectadas en el sentido de que la relación entre las partes es la condición de existencia e identidad del estado complejo. En cambio, en la mera suma de partes la forma cómo las partes están conectadas no es esencial. Así, la mera suma deja de existir si una de sus partes deja de existir mientras que un estado complejo deja de existir si la relación entre sus partes es lo que cambia (entonces, puede dejar de existir sin que ninguna de sus partes deje de existir). De manera que si las partes de $\mathrm{X}$ se conservan, pero $\mathrm{X}$ deja de existir porque la relación entre ellas cambia, $\mathrm{X}$ es un complejo.

En párrafos anteriores se decía que, si se tiene una experiencia de rojo, habrá una conciencia focal externa de la rojez, pero, a la vez, habrá una conciencia periférica interna de la experiencia misma, Entonces ¿cuál es la relación específica que establecen sus partes para que haya conciencia focal externa y, a la vez, conciencia periférica interna? Para poder responder la pregunta anterior se introduce la distinción entre representación directa y representación indirecta. Hay un sentido en el cual una representación directa de una parte de algo puede representar indirectamente a todo 
ese algo. Por ejemplo, la representación directa de la superficie de una fruta puede representar indirectamente a toda la fruta, o alguien puede decir que le duele el cuerpo en virtud de que le duele el estómago. Ahora bien, lo que permite, para seguir con el ejemplo, que la superficie de la fruta represente indirectamente a toda la fruta es que la superficie de la fruta y la fruta se encuentran relacionadas de una manera íntima, profunda y cohesiva. En cambio, esa relación no se da entre la fruta y el lugar donde está contenida. Para el caso de la experiencia consciente diremos lo mismo: tiene que existir una relación de parte tal que permita que la auto-conciencia no-posicional representando directamente a la conciencia posicional represente indirectamente a la conciencia pre-reflexiva. La relación de parte que se necesita para que la auto-conciencia no-posicional representando directamente a la conciencia posicional represente indirectamente a la conciencia pre-reflexiva es la relación de constituir un estado complejo, no una mera suma de partes. Entonces mereológicamente la conciencia pre-reflexiva es un estado complejo de partes. Pues por lo anterior la autoconciencia no-posicional y la conciencia posicional, que son las partes que componen la conciencia pre-reflexiva, se encuentran relacionadas de una manera esencial tal que esa relación es la condición de existencia e identidad de la propia conciencia pre-reflexiva. No es, por tanto, una mera suma de partes.

Para que pueda darse la representación directa y la representación indirecta en la conciencia pre-reflexiva, ésta tiene que ser, como ya se vio, un estado complejo de partes en que las partes (la autoconciencia no-posicional y la conciencia posicional) se encuentren relacionadas de una manera interna, constitutiva y esencial, y no como estados ontológicamente independientes el uno del otro. Es bajo este tipo de mereología que puede darse la representación indirecta de un estado mental consciente mediante la representación directa de una de sus partes. En el caso de la conciencia pre-reflexiva, ésta se representa indirectamente a sí misma mediante la autoconciencia no-posicional que representa directamente la conciencia posicional. La autoconciencia no-posicional representa la conciencia posicional porque establece una relación interna, constitutiva y esencial con ésta, la que a su vez establece una relación de parte relevante con la conciencia pre-reflexiva. Es de este modo que la autoconciencia no-posicional es la conciencia implícita que acompaña a toda conciencia posicional. El carácter subjetivo de la conciencia se manifestaría, entonces, fenomenológicamente hablando, como conciencia implícita. Por ejemplo, en el momento pre-reflexivo de la experiencia de mirar un castaño, se está posicionalmente consciente (lo que podría considerarse a su vez como conciencia focal externa) del castaño y, simultáneamente, se está no-posicionalmente consciente, es decir, implícitamente consciente (lo que podría considerarse a su vez como conciencia periférica interna) de la experiencia misma. Con esto también se apunta a una distinción de grado entre la conciencia del objeto y la conciencia de la experiencia. Así, no parece erróneo decir que la representación indirecta se manifestaría fenoménicamente como autoconciencia no-posicional/ conciencia periférica interna/conciencia implícita y la representación directa se manifestaría fenoménicamente como conciencia posicional/conciencia focal externa/conciencia explícita.

El carácter subjetivo debe entenderse en términos de autoconciencia no-posicional. Como se dijo anteriormente, si la autoconciencia no-posicional debe considerarse de modo intrínseco, entonces la conciencia pre-reflexiva es auto-representacional. Dado que la autoconciencia no-posicional que representa la conciencia posicional es parte de la conciencia pre-reflexiva, puede decirse que la conciencia pre-reflexiva se representa a sí misma. Siguiendo la propuesta auto-representacional, tenemos que la conciencia pre-reflexiva (es decir, un estado mental consciente) es un estado complejo compuesto de la conciencia posicional (que es una parte apropiada de ese estado) y de la autoconciencia no-posicional (que es otra parte apropiada de ese estado), donde la autoconciencia no-posicional representa la conciencia pre-reflexiva por representar la conciencia posicional.

\section{IDENTIDAD EXPERIENCIA/ SUJETO DE EXPERIENCIA}

Una vez introducidos los elementos auto-representacionales, ahora es el turno de desarrollar la idea de que habría una identidad entre una experiencia y el sujeto de esa experiencia. Para ello es necesario, primero, desplegar el aparataje conceptual de Galen Strawson con respecto a la idea de sujeto de experiencia. Tal aparataje nos permitirá establecer la diferencia entre ser un Ego/ Yo y ser un sujeto de experiencia. 
Empecemos entonces estableciendo qué es, para Strawson, la Tesis de Frege. Ésta dice: "No puede haber experiencia sin un sujeto de experiencia porque la experiencia es necesariamente para alguien o algo, un sujeto experimentador. La experiencia implica necesariamente una dimensión cualitativa, y esa dimensión cualitativa es necesariamente para alguien o algo" (Strawson, 1997, p. 148). O en palabras de Frege: "Una sensación no es posible sin alguien que la sienta" (Frege, 1998, p. 209). Parece que la Tesis de Frege es una verdad metafísica e incluso hasta podría considerarse que es una obviedad conceptual. Dado que según el concepto mismo de experiencia hay algo que es cómo es tener una experiencia, se sigue necesariamente que una experiencia es para alguien ( $o$ algo). Pero si una experiencia es tal que hay algo que es cómo es ser para alguien o algo tener esa experiencia, entonces no puede haber experiencia sin un sujeto de experiencia.

Siendo que hay un sujeto de experiencia, parece pertinente preguntarse por la naturaleza de éste, así como también es pertinente la pregunta por la relación entre una experiencia y el sujeto de esa experiencia. Para lograr responder a estas preguntas es que se presentará la concepción reducida (thin conception) de sujeto de experiencia ${ }^{11}$.

La concepción reducida sostiene que el sujeto de experiencia es algo que existe si y solo si existe la experiencia de la cual es el sujeto. De manera que un sujeto de experiencia no existe ni puede existir en cualquier momento determinado a menos que tenga una experiencia en ese momento. Así, bajo la concepción reducida un sujeto de experiencia es "esencialmente experiencialmente 'vivo', no puede ser meramente disposicional; si no hay experiencia (...), no hay sujeto de experiencia." (Strawson, 2011, pp. 260-261). Con lo cual el sujeto de experiencia no continúa existiendo si no existe experiencia alguna. El sujeto de experiencia solo existe cuando una experiencia existe. No existe ni antes ni después de tener alguna experiencia. En ese sentido el sujeto de experiencia es necesariamente un sujeto vivo (live subject).

Ahora bien, aunque bajo la concepción reducida el sujeto de experiencia y su experiencia existen necesariamente al mismo tiempo, nada implica esto sobre la relación entre ambos. Aun cuando el sujeto de experiencia se presente necesariamente al mismo tiempo que su experiencia, perfectamente podría ser extrínseco respecto a ella. Pero también podría tratarse de una relación intrínseca, pues podría ser que una experiencia y su sujeto estén en un tipo de relación que hace que se presenten como una unidad, y que esa sea la razón por la que se presenten necesariamente al mismo tiempo. Inmediatamente Strawson establece que la relación entre un sujeto y la experiencia de ese sujeto es de unidad. De hecho, aunque no haya implicación, la concepción reducida del sujeto de experiencia parece estar más cercana a la idea de una relación como unidad que de otra cosa. Esto se debe a que si sujeto de experiencia es algo que existe si y solo si existe la experiencia de la cual es el sujeto, de manera que no existe ni puede existir en cualquier momento determinado a menos que tenga una experiencia en ese momento, entonces parece que una explicación de ese hecho es pensar la relación entre sujeto y experiencia como una unidad.

Teniendo en cuenta la Tesis de Frege y la concepción reducida del sujeto de experiencia, Strawson propone que la relación de unidad entre sujeto y experiencia es fuerte (2010, p. 69). Aun cuando la experiencia o el campo experiencial es amplio (involucra muchas cosas, un contenido, áreas focales, áreas periféricas, entre otras cosas), la experiencia se presenta de manera unificada y, por lo tanto, indivisible. Pero es unificada e indivisible por ser la experiencia de un solo sujeto de experiencia en un momento dado. Con lo cual se muestra que existe una codependencia entre una experiencia o, más precisamente, entre el campo experiencial y el sujeto de esa experiencia:

un campo, un único campo [experiencial], implica un sujeto, un único sujeto [de experiencia], y a la inversa. Si traes a la existencia a un único sujeto reducido [thin subject] en $t l$ entonces necesariamente traes a la existencia a un único campo experiencial unificado en $t 1$, y a la inversa. La unidad del sujeto (reducido) en $t 1$ implica, y es esencialmente constitutiva de, la unidad de su campo experiencial total en $t 1$. Así también la unidad del campo experiencial total en $t 1$ implica la unidad de su sujeto en $t 1$, pues el material que es experienciado es necesariamente experienciado en una única o unificada perspectiva experiencial. (Strawson, 2010, p. 81). 
La unidad fuerte, que viene dada por la codependencia entre un sujeto y su experiencia, consiste por tanto en que para un único campo experiencial total hay un único sujeto reducido, y que para único un sujeto reducido hay un único campo experiencial total; sin importar su complejidad o simpleza, o su duración, un campo experiencial total siempre implicará un único sujeto reducido.

La unidad formada por el campo experiencial y el sujeto de experiencia, como unidad indivisible, es una "unidad concreta (...) de un muy fuerte tipo." (Strawson, 2010, p. 84). A diferencia de objetos físicos como mesas, tenedores y otros, los cuales pueden perder una de sus partes y todavía considerarse los objetos físicos que son (lo cual quiere decir que tienen condiciones de identidad relajadas), la unidad fuerte entre un sujeto y su campo experiencial implica que no se puede perder ninguna de sus partes y seguir siendo lo que es (lo cual quiere decir que tienen condiciones de identidad estrictas).

La experiencia considerada como tal tiene que estar compuesta del campo experiencial y del sujeto de experiencia, con lo cual la experiencia debe tener partes o elementos distinguibles. Pero por ser una unidad fuerte tales partes distinguibles tienen que mantenerse juntas en una relación específica tal, que si ésta es cambiada de la forma que sea ya no se tendrá más la misma experiencia. El campo experiencial, entonces, no podría existir aparte del sujeto de experiencia, y viceversa. La unidad es una relación fuerte tal que, además de tener que mantenerse de una determinada manera, hace que el

[campo experiencial] está, de alguna manera, implicado en [el sujeto de experiencia] de tal modo que su ser es al menos en parte constitutivo del ser del [sujeto de experiencia]. En esta visión [el campo experiencial] es, por así decirlo, el cuerpo o la carne del [sujeto de experiencia], sin el cual el [sujeto de experiencia] (como sujeto reducido) no puede existir, porque entonces no sería nada. El [sujeto de experiencia], sentimos, (...) no es nada sin el [campo experiencial] —no solo completamente vacío- sino que inexistente. El [sujeto de experiencia], en esta visión, posiblemente no puede existir aparte del [campo experiencial]. (Strawson, 2008b, p. 169, [cursivas propias]).
Entonces, se tiene que la existencia de la experiencia no es realmente otra cosa más que la existencia del campo experiencial y del sujeto de experiencia. La experiencia está compuesta del campo experiencial y del sujeto de experiencia. Es decir, la experiencia es idéntica al campo experiencial más el sujeto de experiencia.

Para poder desarrollar de mejor manera la idea anterior, por ahora dejaremos en stand-by el desarrollo conceptual de Strawson e introduciremos la tesis de composición como identidad.

La composición como identidad es una tesis ontológica-mereológica acerca de la relación entre un objeto concreto y las partes que lo componen. La composición como identidad puede ser definida del siguiente modo: "el todo es literalmente idéntico a todas sus partes." (Wallace, 2011a, 2011b). Otro modo de definirla es diciendo que el "predicado 'son' usado para indicar la relación de composición es literalmente otra forma del 'es' de identidad." (Wallace, 2011a, 2011b). En el caso de la experiencia, de la primera definición se sigue que una experiencia es literalmente idéntica al campo experiencial y al sujeto de experiencia. De la segunda definición se sigue que el campo experiencia y el sujeto de experiencia son idénticos a la experiencia.

Es importante aclarar que la composición como identidad "no significa que el todo es idéntico a cada una de sus partes, tomadas individualmente. Si no más bien, el todo es idéntico a sus partes tomadas conjuntamente." (Wallace, 2011a, 2011b). O, más específicamente, la composición como identidad no significa que el todo es idéntico a cada una de sus partes consideradas distributivamente. Si no más bien, significa que el todo es idéntico a sus partes consideradas colectivamente $^{12}$. Entonces, una experiencia es idéntica al campo experiencial y al sujeto de experiencia considerados colectivamente. Para poder hacer sentido a la tesis de composición como identidad y a la diferencia entre considerar las partes distributiva y colectivamente es necesario dar "sentido gramatical de la identidad muchos-uno" (Wallace, 2011a, 2011b).

Para lograr lo anterior es que se debe adoptar un lenguaje plural completo con términos, variables, cuantificadores y predicados, todos plurales, e introducir un predicado híbrido de identidad singular-plural. Para los términos plurales se puede usar ““,' como una forma de 
ir concatenando términos singulares, donde, por ejemplo ' $\mathrm{x}, \mathrm{y}$ ' significa ' $\mathrm{x}$ e $\mathrm{y}$, considerados juntos'.” (Wallace, 2011a, 2011b). O, siguiendo con nuestro caso, "campo experiencial, sujeto de experiencia' significa 'campo experiencial y sujeto de experiencia, considerados colectivamente'. Sider dice: "En este nuevo lenguaje, los predicados-argumentos no son de manera fija singulares o plurales; de hecho, uno puede simplemente dejar de lado la distinción singular-plural por completo" (2007, p. 57). Con lo cual el lenguaje plural sería el más capacitado para dar cuenta del hecho de que 'experiencia' y 'campo experiencial, sujeto de experiencia' "seleccionan la misma porción de realidad." (Sider, 2007, p. 57). Con respecto al predicado híbrido de identidad singular-plural, éste se usa porque el "predicado tradicional de primer orden de identidad solo acepta términos singulares como lugar de argumentos." (Wallace, 2011a, 2011b). Pero al usarse el predicado híbrido de identidad se permite a términos plurales como lugares de argumento. Es importante aclarar que el predicado híbrido de identidad no entra en contradicción con el predicado tradicional de primer orden de identidad, sino que ahora este último pasaría a ser un caso del primero. Así, desde la composición como identidad se puede representar el predicado híbrido de identidad como " "=h", que toma ya sea términos plurales o términos singulares como lugares de argumento: $\mathrm{a}=\mathrm{h} \mathrm{b}$, donde $\mathrm{a} \mathrm{y} \mathrm{b}$ pueden ser tanto términos plurales como términos singulares" (Wallace, 2011a, 2011b).

Por último, basados en el análisis anterior parece ser claro que la composición como identidad se conjuga de buena manera con lo que hemos dicho hasta aquí para llegar a la idea de identidad entre la experiencia y el sujeto de experiencia. Esto puede verse, por ejemplo, en que es posible relacionar la composición como identidad con la idea de auto-representación. En vista a que todo episodio de conciencia se representa a sí mismo, puede decirse que la conciencia se conecta a sí misma. Lo cual es indicativo de la composición como identidad en tato "conecta una cosa a sí misma" (Sider, 2007, p. 54). También puede verse que si la composición como identidad solo aplica cuando se considera que el todo es idéntico a sus partes tomadas colectivamente, y no distributivamente, esta idea de composición encaja con las condiciones de identidad de la conciencia. Pues, las partes del todo deben considerarse de cierta manera conjunta para que sean idénticas al todo, como ocurre en el caso de la conciencia. Las partes de la conciencia deben encontrarse en una relación tal que solo mediante esa relación son constituyentes de ella. La conciencia es un estado complejo de partes porque sus partes están esencialmente interconectadas en el sentido de que la relación entre éstas es la condición de existencia e identidad de la conciencia. Precisamente, las partes de una unidad fuerte han de mantenerse juntas en una relación específica tal que, si ésta es cambiada no se tendrá más la misma conciencia. Entonces, el considerar las partes colectivamente puede ser entendido como una condición de identidad similar a la que es propia de un estado complejo de partes y una unidad fuerte.

Pues bien, las ideas de unidad fuerte y de composición como identidad nos permiten poner la relación entre una experiencia y su sujeto como si ambos términos de la relación sólo fueran conceptualmente distintos, no realmente distintos. A esta altura es necesario oponer la distinción real a la distinción conceptual, para poder dar con el punto que queremos establecer. Por distinción real se entiende la distinción que puede realizarse entre dos (o más) cosas en la realidad: que dos (o más) cosas en la realidad son realmente distintas significa que pueden existir aparte una(s) de la(s) otra(s). La distinción real aplica a dos (o más) cosas si pueden "existir aparte "fuera de nuestro pensamiento', como opuesto a meramente 'en nuestro pensamiento"' (Strawson, 2008a, p. 271). Un ejemplo de distinción real es el que se da entre dos o más substancias ${ }^{13}$. Para entender qué es la distinción conceptual se recurrirá al siguiente ejemplo, dado por Strawson: se pide que se considere la triangularidad y la trilateralidad de una figura rectilínea cerrada (un triángulo hecho de madera, por ejemplo). Parece obvio que existe una distinción entre la triangularidad y la trilateralidad. La primera es sobre ángulos y la segunda es sobre lados. Pero, aunque exista una distinción entre ambas, ningún objeto puede instanciar una sin instanciar la otra. Ambas propiedades pueden existir aparte en nuestro pensamiento, pero no fuera de éste: la triangularidad y la trilateralidad pueden ser distinguidos en el pensamiento, pero no pueden existir aparte en la realidad concreta. Pues, "cualquier caso actualmente existente de triangularidad es [un] caso [actualmente existente] de trilateralidad" (Strawson, 2008b, p. 180). 
En efecto, "la real existencia de una consiste enteramente en la misma cosa en la cual consiste enteramente la real existencia de la otra. Ni la existencia real de la una excede la existencia real de la otra de manera alguna. El ser (concreto) de una es - (...) - el ser (concreto) de la otra (...)" (Strawson, 2008a, p. 271). Por el ser (concreto) hay que entender el triángulo hecho de madera del ejemplo. Así, cuando hay un triángulo hecho de madera necesariamente hay triangularidad y necesariamente hay trilateralidad. El ser (concreto) de estas propiedades es el mismo. Por lo tanto, dado que no pueden existir la una aparte de la otra, lo que hay entre ellas es una distinción conceptual. Una vez entendida la relación entre una experiencia y su sujeto en términos de unidad fuerte y de composición como identidad, se sigue que solo se le podría aplicar la distinción conceptual. Esto es así porque, como vimos, la experiencia es una unidad fuerte que se mantiene siendo lo que es sí y solo si sus partes componentes (el campo experiencial y el sujeto de experiencia) están esencialmente interconectadas en una relación específica tal que la experiencia es el campo experiencial y el sujeto de experiencia o, al revés, el campo experiencial y el sujeto de experiencia son la experiencia. Por ser una unidad fuerte la experiencia es idéntica a sus componentes. Entonces, los términos 'experiencia' y 'campo experiencial, sujeto de experiencia' seleccionan la misma porción de realidad. Un caso actualmente existente de experiencia es un caso actualmente existente de sujeto de experiencia. Siendo que el ser concreto del sujeto de experiencia es la experiencia misma, en cualquier caso concreto en que haya una experiencia necesariamente hay un sujeto de experiencia. La existencia real del sujeto de experiencia consiste enteramente en la misma cosa en la cual consiste enteramente la existencia real de la experiencia. La existencia real de la experiencia no excede la existencia real del sujeto de experiencia. Lo anterior puede plantearse diciendo que la experiencia "(...) no puede venir aparte de sí misma." (Strawson, 2008a, p. 272). Esto significa que una experiencia y su sujeto no pueden existir aparte. De este modo, una experiencia y su sujeto no solo "(...) son abstractamente distintos, pero actualmente inseparables." (Martin, 1997, 2003), sino que no "es posible como una cuestión de hecho objetivo" (Strawson, 2008a, p. 271) que existan aparte. Por la unidad fuerte y la composición como identidad no hay distinción real entre una experiencia y su sujeto, dado que no pueden existir separadamente. Entre el sujeto de experiencia y una experiencia solo hay una distinción 'en nuestro pensamiento', no 'fuera de nuestro pensamiento' 14 .

Por último, digamos que, al no haber una distinción real entre una experiencia y su sujeto de experiencia, es posible realizar el paso desde la unidad fuerte y la composición como identidad a la identidad. Si una experiencia y su sujeto designan la misma porción de realidad de tal modo que no pueden existir aparte, "entonces ellos no son realmente dos sino solo uno: ellos son idénticos (es decir, es — por supuesto-idéntico a sí mismo)" (Strawson, 2008b, p. 181). Strawson se refiere a la no distinción real entre el sujeto y su experiencia como la tesis de la identidad experiencialexperimentador (Experience/Experiencer Identity Thesis), que enuncia del siguiente modo: "el sujeto es idéntico con su experiencia" (2011, p. 260$)^{15}$.

\section{CONCIENCIA}

\section{PRE-REFLEXIVA Y LA TESIS DE LA IDENTIDAD EXPERIENCIA- EXPERIMENTADOR}

Una vez finalizado el despliegue del aparataje conceptual de Strawson con respecto a la idea de sujeto de experiencia, queda por ver cómo tal despliegue nos permite establecer la no necesidad de postular un Ego/Yo, y así introducir la idea de que una teoría no-egológica de la conciencia no excluye la existencia de un sujeto de experiencia porque la experiencia se toma a sí misma como sujeto de experiencia.

La conciencia pre-reflexiva para explicar la Tesis de Frege no tiene la necesidad de postular un Ego/Yo. La conciencia posee una estructura no-egológica: la explicación de que hay algo que es cómo es para alguien o algo tener una experiencia se logra porque la experiencia es capaz de referirse a sí misma. Se refiere a sí misma porque toda conciencia pre-reflexiva (es decir, toda experiencia consciente) es autoconciencia no-posicional de sí misma en la medida en que la autoconciencia no-posicional forma parte del estado mental consciente. Es importante destacar que la auto-referencia del estado mental consciente es algo que se requiere para mantener el carácter subjetivo de los estados mentales conscientes. En efecto, el carácter subjetivo "(...) es un regreso, a 
pesar de todo, a sí mismo" (Sartre, 1967, p. 123). Entonces, el sujeto de experiencia podría ser la autoconciencia no-posicional.

De este modo, si recordamos que en el apartado 3 anterior habíamos dicho que la experiencia está compuesta del campo experiencial y del sujeto de experiencia y que en el apartado 2 habíamos dicho que la conciencia pre-reflexiva está compuesta de la autoconciencia no-posicional y la conciencia posicional, entonces podemos establecer las siguientes equivalencias: experiencia $=$ conciencia pre-reflexiva, campo experiencial $=$ conciencia posicional $\mathrm{y}$ sujeto de experiencia $=$ autoconciencia no-posicional.

Por lo tanto, para defender la tesis de la identidad experiencia/experimentador necesario ver cómo puede entenderse la conciencia pre-reflexiva como una unidad fuerte en conjunción con la composición como identidad, y finalmente como una identidad. Primero, dado que anteriormente definimos a la conciencia pre-reflexiva como un estado complejo de partes, entonces no parece errado ahora tratar a ésta, además, como una unidad fuerte. Tanto la unidad fuerte como el estado complejo de partes se definen por una relación específica entre sus partes: las partes de la unidad fuerte y del estado complejo tienen que mantenerse juntas en una relación específica tal que, si ésta es cambiada de la forma que sea, ya no existirán más. La unidad fuerte y el estado complejo de partes no pueden perder ninguna de sus partes y seguir siendo lo que son. Siendo así una unidad fuerte, la conciencia pre-reflexiva no podría existir sin que la autoconciencia no-posicional y la conciencia posicional, que son sus partes, se encuentren en la relación adecuada. Es fácil ver ahora, en segundo lugar, que es posible aplicar la tesis de la composición como identidad a la conciencia pre-reflexiva. La composición como identidad aplica porque la conciencia pre-reflexiva es idéntica a sus partes tomadas colectivamente y no distributivamente. En particular, la autoconciencia no-posicional y la conciencia posicional deben considerarse de cierta manera para que sean idénticas a la conciencia pre-reflexiva. Entonces, la composición como identidad es similar a la unidad fuerte en tanto que las partes de la conciencia pre-reflexiva deben encontrarse en una relación tal que solo en esa relación son constituyentes de ella. Esa relación es la relación de auto-representacionalidad. Solo de este modo la conciencia pre-reflexiva es idéntica a la autoconciencia no-posicional y a la conciencia posicional, o la autoconciencia no-posicional y la conciencia posicional son idénticas a la conciencia pre-reflexiva.

Ahora es posible decir que la relación entre la conciencia pre-reflexiva y la autoconciencia no-posicional (es decir, la relación entre una experiencia y su sujeto), entendida en términos de unidad fuerte y de composición como identidad, es tal que no se aplica la distinción real. Por ser unidad fuerte y por composición como identidad, la autoconciencia no-posicional y la conciencia posicional están esencial y específicamente relacionadas de manera tal que la autoconciencia no-posicional y la conciencia posicional son la conciencia pre-reflexiva. Entonces, los términos 'autoconciencia no-posicional, conciencia posicional' y 'conciencia pre-reflexiva' seleccionan la misma porción de realidad. Esto implica que la conciencia pre-reflexiva y la autoconciencia no-posicional (es decir, una experiencia y su sujeto) no pueden existir aparte. Es decir, la autoconciencia no-posicional no es ontológicamente independiente con respecto a la conciencia pre-reflexiva. La primera es el ser concreto de la segunda: en lo que consiste la real existencia de la conciencia pre-reflexiva es lo mismo en lo que consiste la real existencia de la autoconciencia no-posicional. Pero, aunque no haya una distinción real entre ambas, puede decirse que sí hay una distinción conceptual. La siguiente cita puede leerse como una confirmación de este punto: dado que la conciencia pre-reflexiva es una relación entonces "implica dualidad, y por lo tanto, separación, por lo menos virtual" (Sartre, 1993, p. 111). O de manera más categórica: dado que entre la autoconciencia no-posicional y la conciencia pre-reflexiva no hay una distinción real (sino conceptual), “(...) si nos preguntamos ahora: ¿qué es lo que separa [a la conciencia pre-reflexiva]?, nos vemos obligados a confesar que no es nada." (Sartre, 1993, p. 111). No parece errado entonces que la idea de separación virtual o de nada sea entendida como mera distinción conceptual (en tanto no puede existir separadamente con respecto a la conciencia pre-reflexiva). Gennaro lo pone del siguiente modo:

(...) la 'dualidad' en el nivel pre-reflexivo (...) no es una 'separación' literal entre (...) la autoconciencia no-posicional y la otra parte del estado complejo consciente [la 
conciencia posicional]. Tal vez esto es lo que [se] entiende por una 'separación virtual;' es decir, una separación dentro de un estado complejo consciente". (2002, p. 315).

Gennaro continúa diciendo que "hablar de una 'dualidad dentro de una unidad' hace sentido (...). También hace sentido la afirmación de que no hay 'nada' entre las partes, ya que son partes de un mismo estado mental consciente" (2002, p. 315).

Lo anterior nos permite pasar ahora a la idea de identidad. Si tanto la existencia de la autoconciencia no-posicional como la existencia de la conciencia posicional no son realmente otra cosa que la existencia de la conciencia pre-reflexiva, es posible decir que están en una relación de identidad. Ahora bien, dado que la conciencia pre-reflexiva puede ser entendida como una identidad, podemos establecer, de manera más detallada, cómo se aplica la tesis de la identidad experiencia/experimentador en una teoría no-egológica de la conciencia que no postula la existencia de un Ego/Yo. En efecto, si la conciencia pre-reflexiva (es decir, la experiencia consciente) es una unidad tal que sus partes componentes no pueden existir separadamente, entonces la autoconciencia no-posicional, que es el sujeto de experiencia, debe entenderse bajo la concepción reducida según la cual el sujeto de experiencia es algo que existe si y solo si existe la experiencia consciente de la cual es el sujeto. El sujeto de experiencia (es decir, la autoconciencia no-posicional) no existe ni puede existir en cualquier tiempo a menos que exista su experiencia (es decir, la conciencia pre-reflexiva). Pero si a causa de ser una unidad fuerte la conciencia pre-reflexiva es entendida también como una identidad, entonces la conciencia pre-reflexiva y su sujeto de experiencia no son realmente distintos, sino que son idénticos. La conciencia pre-reflexiva y su sujeto, la autoconciencia no-posicional, constituyen la misma porción de realidad. Con esto se puede establecer ahora, respecto a la conciencia pre-reflexiva, la tesis de la identidad experiencia/experimentador.

Finalmente, como hemos visto, la autoconciencia no-posicional es solo una distinción conceptual de la conciencia pre-reflexiva, no realmente distinta de ella, tenemos que el sujeto de experiencia no es realmente distinto, sino idéntico, a la conciencia pre-reflexiva. Con lo cual se presenta una tesis más económica. Puesto que, para dar cuenta del sujeto de experiencia, la tesis no-egológica de la conciencia no tiene la necesidad de postular otra entidad diferente del estado mental mismo.

De ahí toman sentido las palabras de Williford, quien en una serie de escritos se refiere a la idea de la tesis de la identidad experiencial experimentador de la siguiente manera: "la auto-representación constituye la subjetividad de la conciencia. Por 'subjetividad' (...) quiero decir el hecho de que todas las experiencias conscientes son para un sujeto. Desde esta perspectiva, el sujeto de conciencia es solo el episodio consciente mismo" (2006, p. 121); "El 'modo de darse en primera persona' [first-personal mode of givenness], como veremos, está bien caracterizado por la auto-manifestación de la corriente [de la conciencia], en términos sartreanos, por la autoconciencia no-posicional" (2011, p. 203); "Toda corriente de la conciencia tiene su sujeto transitorio (a saber, sí mismo) que no es un yo sustancial" (2015, p. 17).

Dado que 'modo de darse en primera persona' indica que en la conciencia hay algo que es cómo es ser para alguien o algo, ese algo o alguien sería la autoconciencia no-posicional como sujeto de experiencia. Siendo solo conceptual y no realmente distinto. 


\section{Referencias}

Block, N. (1995). On a confusion about a function of consciousness. Behavioral and Brain Sciences, 18, 227-247.

Brentano, F. (2009). Psychology from an Empirical Standpoint. London: Routhledge.

Frege, G. (1998). Ensayos de Semántica y Filosofía de la Lógica. Madrid: Tecnos.

Gennaro, R. (2002). Jean-Paul Sartre and the HOT Theory of Consciousness. Canadian Journal of Philosophy, 32(3), 293-330.

Gennaro, R. (2012). The Consciousness Paradox. Consciousness, Concepts, and High-Order Thoughts. Cambridge/London: A Bradford Book The MIT Press.

Janzen, G. (2008). The Reflexive Nature of Consciousness. Amsterdam/Philadelphia: John Benjamins Publishing Company.

Kant, I. (1970). Philosophical Correspondence 1759-1799. Chicago: The University of Chicago Press.

Kriegel, U. (2009). Subjective Consciousness: A SelfRepresentational Theory. New York: Oxford University Press.

Kriegel, U. (2012a). Précis of Subjective Consciousness: A SelfRepresentational Theory. Philos Stud, 159, 443-445.

Kriegel, U. (2012b). In defense of self-representationalism: reply to critics. Philos Stud, 159, 475-484.

Martin, C. B. (1997). On the Need for Properties: The Road to Pythagoreanism and Back. Synthese, 112, 193-231.

Martin, C. B. (2003). Power for Realist. En J. Bacon, K. Campbell, \& Ll. Reinhardt (Eds.), Ontology, Causality and Mind. Essays in Honour of D. M. Armstrong (pp. 175-194). Cambridge: Cambridge University Press.

Nagel, T. (2003). ¿Cómo es ser un murciélago? En M. Ezcurdia \& O. Hansberg (Eds.), La Naturaleza de la Experiencia. Volumen I: Sensaciones (pp. 45-63). México D.F.: Universidad Nacional Autónoma de México.

Nolan, D. (1997). Reductionism and Nominalism in Descartes's Theory of Attributes. Topoi, 16(2), 129-140.

Ortiz, E. (2017). La conciencia como puente temático entre la fenomenología y la filosofía analítica. Mutatis Mutandis: Revista Internacional de Filosofía, 9, 163-197.

Ortiz, E. (2018). Sartre: una teoría auto-representacional de la conciencia. Revista de Humanidades de Valparaíso, 11, 115-137.

Rosenthal, D. M. (2012). Awareness and Identification of Self. En J. Liu \& J. Perry (Eds.), Consciousness and the Self: New Essays (pp. 22-50). Cambridge: Cambridge University Press.

Rowlands, M. (2011). Jean-Paul Sartre's Being and Nothingness. Topoi, 30, 175-180.

Sartre, J. P. (1967). Consciousness of Self and Knowledge of Self. En N. Lawrence \& D. O'Connor (Eds.), Readings in Existential Phenomenology (pp. 113-142). New Jersey: Prentice-Hall.

Sartre, J. P. (1968). La trascendencia del Ego. Buenos Aires: Ediciones Calden.

Sartre, J. P. (1993). El Ser y la Nada. Barcelona: Altaya.

Sebastián, M. Á. (2012a). Experimental Awareness: Do You Prefer "It" To "Me"? Philosophical Topics, 40(2), 155-177.
Sebastián, M.Á. (2012b). Uriah Kriegel Subjective Consciousness: A Self-Representational Theory. Disputatio, IV(32), 413-417.

Sider, T. (2007). Parthood. Philosophical Review, 116(1), 51-91. Spinoza, B. (2009). Ética. Madrid: Alianza Editorial.

Strawson, G. (1997). La Realidad Mental. Barcelona: Editorial Prensa Ibérica.

Strawson, G. (1999). The Self and the SESMET. Journal of Consciousness Studies, 6(4), 99-135.

Strawson, G. (2008a). The Identity of the Categorical and the Dispositional. Analysis, 68(4), 271-282.

Strawson, G. (2008b). What is the Relation Between an Experience, the Subject of the Experience, and the Content of the Experience? En G. Strawson, Real Materialism (pp. 151187). Oxford: Oxford University Press.

Strawson, G. (2010). Fundamental Singleness: How to Turn the 2nd Paralogism into a Valid Argument. En P. Basile, J. Kiverstein, \& P. D. Phemister (Eds.), The Metaphysics of Consciousness. Royal Institute of Philosophy Supplement, 67 (pp. 61-92). Cambridge: Cambridge University Press.

Strawson, G. (2011). The Minimal Subject. En S. Gallagher (Ed.), The Oxford Handbook of the Self (pp. 253-278). Oxford: Oxford University Press.

Wallace, M. (2011a). Composition as Identity: Part 1. Philosophy Compass, 6(11), 804-816.

Wallace, M. (2011b). Composition as Identity: Part 2. Philosophy Compass, 6(11), 817-827.

Williford, K. (2006). The Self-Representational Structure of Consciousness. En U. Kriegel \& K. Williford (Eds.), SelfRepresentational Approaches to Consciousness (pp. 111-142). Cambridge/London: A Bradford Book The MIT Press.

Williford, K. (2011). Pre-Reflective Self-Consciousness and the Autobiographical Ego. En J. Webber (Ed.), Reading Sartre. On Phenomenology and Existentialism (pp. 195-210). London/ New York: Routledge.

Williford, K. (2015). Representationalisms, Subjetive Character, and Self-Acquaintance. En T. Metzinger \& J. Windt (Eds.), Open MIND (pp. 1-27). Frankfurt am Main: MIND group.

Zahavi, D. (2000). Self and Consciousness. En D. Zahavi (Ed.), Exploring the Self. Philosophical and Psychopathological Perspectives on Self-Experience (pp. 55-74). Oxford: Oxford University Press.

Zahavi, D. (2005). Subjectivity and Selfhood. Investigating the First-Person Perspective. Cambridge/London: A Bradford Book The MIT Press.

Zahavi, D. (2007). Subjectivity and the first-person perspective. Southern Journal of Philosophy, 45, 66-84.

Zahavi, D. (2018). Consciousness, self-consciousness, selfhood: A reply to some critics. Review of Philosophy and Psychology, 9, 703-718. 


\section{Notas al final}

1 Sobre la conciencia fenoménica, véase: Nagel (2003) y Block (1995).

2 M. A. Sebastián se refiere a estas distintas funciones del Ego/Yo como función semántica (el Ego/Yo está presente en la conciencia como contenido de la experiencia) y función meta-semántica (el Ego/Yo da cuenta del contenido de la experiencia). Específicamente, la función semántica hace referencia al contenido de la experiencia. En cambio, la función meta-semántica hace referencia a lo que se requiere para dar cuenta de dicho contenido Esto es todo lo dicho por Sebastián. Lamentamos que el autor no desarrolle lo anterior en detalle.

3 “(...) cuando yo estoy preocupado de la realidad exterior (...) yo aún soy (...) consciente de mí mismo ('Yo') como estando en un estado mental consciente. (...) Así [está] la conciencia (...) dirigida al mundo y una conciencia reflexiva [que] se dirige a uno mismo, pero, incluso en el plano irreflexivo, uno todavía tiene (...) pensamientos sobre uno mismo." (Gennaro, 2002, p. 326). Otro ejemplo es Rosenthal (2012).

4 Si bien iniciamos hablando de conciencia fenoménica, ésta y la conciencia pre-reflexiva son equivalentes. También, y como se hará más adelante, es posible usar el vocablo "experiencia" para referirse a la conciencia fenoménica y a la conciencia pre-reflexiva.

La conciencia fenoménica y la conciencia pre-reflexiva son equivalentes porque ambas enfatizan el aspecto perspectival de una experiencia. Para la conciencia fenoménica se trata de que hay algo que es cómo es ser para un sujeto tener una determinada experiencia, es decir, toda experiencia no sólo ocurre en él sino también para él, en el sentido de que el sujeto tiene que ser consciente de esa experiencia como suya. Pero que un estado mental consciente ocurra para un sujeto implica que el estado mental consciente se da bajo cierta perspectiva, es decir, bajo su perspectiva. Del mismo modo, la conciencia pre-reflexiva, que es un aspecto constante y estructural de los estados mentales conscientes, no se refiere al qué de las experiencias sino al cómo de éstas. El cómo se expresa en que la experiencia ocurre para el sujeto como su experiencia. Con esto, las experiencias se sienten de una determinada manera para el sujeto que las tiene. Poseen un gusto subjetivo. (Ortiz, 2017, pp. 166 - 175)

De este modo, aun cuando el término "conciencia", dado el contexto fenomenológico en el cual se encuentra la teoría de la conciencia pre-reflexiva, significa más que nada representación. A diferencia del significado que tiene en el debate actual de la filosofía de la conciencia, es decir, el carácter fenoménico de los estados mentales conscientes. Lo anteriormente comentado nos permite la posibilidad de subsanar esta diferencia terminológica. A este respecto Janzen dice que "todos los estados conscientes tienen intencionalidad (es decir, son de o 'sobre' algo)." (2008, p. 3). Bajo este contexto, "ser fenoménicamente consciente de x" equivale a "representarse a x".

Es decir, "ser consciente de algo es una cuestión de representarlo" (Sebastián, 2012b, p. 2), es muy probable que algún tipo de representación sea necesaria para la conciencia fenoménica.

$5 \quad$ Se usarán indistintamente.

$6 \quad$ Implícita o periférica pueden usarse indistintamente.

$7 \quad$ Si bien el anterior análisis de la conciencia pre-reflexiva está basado en Sartre, es menester aclarar que no es del todo original porque es Brentano el pensador que hizo los primeros análisis de la conciencia que concluían que ésta está dirigida a algo y, a su vez, dirigida a sí misma:

"La presentación del sonido y la presentación de la presentación del sonido forman un único fenómeno mental; es solo por considerarlo en su relación con dos objetos diferentes, uno de los cuales es un fenómeno físico y el otro un fenómeno mental, que decidimos dividirlo conceptualmente en dos presentaciones. En el mismo fenómeno mental en el cual el sonido se presenta a nuestras mentes, simultáneamente aprehendemos el fenómeno metal en sí mismo. Lo que es más, lo aprehendemos de acuerdo a su naturaleza dual en cuanto tiene al sonido como contenido dentro de él, y a él mismo como contenido al mismo tiempo." (2009, p.98).

"Todo acto mental consciente incluye dentro de él una conciencia de sí mismo. Por lo tanto, todo acto mental consciente, no importa lo simple que sea, tiene un doble objeto, un objeto primario y un objeto secundario. El acto más simple, por ejemplo, el acto de oír, tiene como objeto primario al sonido y objeto secundario a sí mismo, el fenómeno mental en el que el

sonido es oído." (Brentano, 2009, p. 119).

$8 \quad$ Para complementar la anterior caracterización de la conciencia pre-reflexiva, véase Rowlands (2011).

9 Para más detalle de la conexión entre intrínsecalidad y auto-representacionalidad, véase Ortiz (2018).

10 La conciencia periférica es un modo en cómo se presenta la intensidad fenoménica. El otro modo es la conciencia focal. La intensidad fenoménica se nota en tanto que algunos ítems en la experiencia consciente son más pronunciados que otros. Por ejemplo, al leer mi conciencia visual del libro es focal mientras mi conciencia visual del escritorio donde se encuentra el libro es periférica.

11 Existen otras concepciones de sujeto de experiencia. La concepción amplia (thick conception) y la concepción tradicional interna (traditional inner conception). Pero para nuestros objetivos no son importantes. De todos modos dejamos unas mínimas definiciones. Por la primera se tiene que el sujeto de experiencia es el organismo entendido como un todo, es decir, bajo esta concepción el sujeto de experiencia es un ser humano o, por ejemplo, un murciélago. Por la segunda se entiende al sujeto de experiencia como una entidad persistente, que es una especie de presencia mental interna persistente distinta del ser humano o del murciélago.

$12 \quad$ Sider lo pone del siguiente modo: “'Ted = cabeza, brazos, piernas, y torso' no significa 'Ted = cabeza y Ted = brazos y Ted $=$ piernas y Ted $=$ torso '. (Eso sería un sin sentido.) Comparen el predicado 'rodeaban' en 'Tom, Dick, and Harry rodeaban al 
preso fugado'. Dos puntos. Primero, este uso de 'rodeaban' es colectivo más que distributivo: la oración no es equivalente a 'Tom rodeaba al preso fugado y Dick rodeaba al preso fugado y Harry rodeaba al preso fugado'. Tom, Dick, y Harry pueden rodear al preso a pesar de que ninguno (...) rodea al prisionero por su cuenta. Segundo, rodeando no es (...) una relación entre el prisionero y alguna entidad extra que envuelve a Tom, Dick, y Harry en uno. (....). Así, 'rodean' es irreductiblemente plural: relaciona a los tres hombres directamente con el prisionero, y es colectiva. Lo mismo sucede con 'es idéntico a', (...) —incluso cuando ese predicado expresa identidad en el sentido más estricto. El signo de identidad en 'Ted = cabeza, brazos, piernas, y torso' es colectivo, y relaciona cabeza, brazos, piernas, y torso directamente con Ted" (2007, p. 56).

13 La distinción real aplicaría en el caso de la substancia porque una substancia es "(...) aquello que es en sí y se concibe por sí, esto es, aquello cuyo concepto, para formarse, no precisa del concepto de otra cosa.” (Spinoza 2009, p. 46). Véase también Nolan (1997).

14 Podría pensarse que, al no haber distinción real entre una experiencia y su sujeto, no caen en el mismo tipo de relación que se establece entre un objeto y sus propiedades. Entre ambos no habría el contraste, que generalmente se considera, entre un objeto y sus propiedades. En efecto, generalmente se considera que un objeto y sus propiedades pueden existir realmente separados. Con lo cual se piensa que existe una distinción real entre ambos, y de esta manera sería posible distinguir "entre la existencia (...) del objeto, dado cualquier tiempo, y su naturaleza, (...) dado cualquier tiempo.” (Strawson, 2008b, p. 171). Pero, ¿es esto así? Basta con observar cualquier objeto, por ejemplo, una mesa, para dar cuenta que lo que generalmente se considera como relación entre un objeto y sus propiedades colapsa en sí misma. En efecto, entre una mesa y sus propiedades "no hay subordinación ontológica de las [propiedades de la mesa] a la [mesa] en sí misma. No hay desigualdad existencial o prioridad de cualquier tipo, no hay dependencia existencial ni del uno ni del otro, ni independencia existencial ni del uno ni del otro.” (Strawson, 2008a, p. 279). ¿Podrían el color de la mesa, la altura de ésta o su extensión existir separados de la mesa? Como la respuesta parece ser negativa, debe concluirse que los objetos y sus propiedades no pueden existir realmente separados. Entonces, entre un objeto considerado en cualquier tiempo y sus propiedades consideradas en ese mismo tiempo, la distinción que se establece entre ellos solo es conceptual, no real. Por lo tanto, no hay problema en ver la relación entre la experiencia y el sujeto de esa experiencia como un caso del tipo de relación esclarecida que se establece entre un objeto y sus propiedades.

15 Esto no es algo nuevo, ya fue propuesto por Kant, quien lo pone del siguiente modo: “( ...) la existencia del pensamiento y la existencia [del sujeto de experiencia] son uno y el mismo." (1970, p. 75).

\section{Agradecimientos}

A Javier Vidal, Sebastián Briceño y al arbitraje anónimo de la Revista Límite, de cuyos comentarios se ha beneficiado enormemente este trabajo. 